\title{
Comprehensive study on manifestation, management and outcome of post COVID rhino orbito cerebral mucormycosis in our institution
}

\author{
Shanthimalar R. ${ }^{1}$, Muthuchitra S. ${ }^{2}$, Mary Nirmala S. ${ }^{2}$, Thamizharasan P. ${ }^{2}$, \\ Udhayachandrika G. ${ }^{2}$, Balaji C. ${ }^{2}$, Indu S. P. ${ }^{1}$, Kowsalya G. ${ }^{1}$, Senthoorani A. ${ }^{1}$, Yuvaraj E. ${ }^{1}$
}

${ }^{1}$ Government Kilpauk Medical College, Chennai, Tamil Nadu, India

${ }^{2}$ Department of ENT, Government Kilpauk Medical College, Chennai, Tamil Nadu, India

Received: 25 September 2021

Revised: 05 October 2021

Accepted: 06 October 2021

\author{
*Correspondence: \\ Dr. Thamizharasan P., \\ E-mail: thamizhent06@gmail.com
}

Copyright: (C) the author(s), publisher and licensee Medip Academy. This is an open-access article distributed under the terms of the Creative Commons Attribution Non-Commercial License, which permits unrestricted non-commercial use, distribution, and reproduction in any medium, provided the original work is properly cited.

\begin{abstract}
Rhino orbital mucor mycosis in covid infected patients presented a challenge in management. We tried analysing comprehensively the manifestations and management and prognosis of post COVID rhino orbito cerebral mucormycosis (ROCM) patients in our institution. Patients with symptoms of ROCM were managed according to the guidelines issued by the directorate of medical education, Tamil Nadu, India. 30 possible ROCM patients were treated so far from May 2021. Patients underwent radiological, microbiological and pathological investigation. In stage 1, nasal cavity alone was involved in 11 patients. In stage 2, paranasal sinus extension was seen in 8 patients. In stage 3 , orbital extension was seen in 10 patients. In stage 4, intra cranial extension was seen in 1 patient. 10 patients were probable ROCM without microbiological or pathological evidence. 20 patients had proven ROCM. They were treated with endoscopic debridement. Out of them 10 patients had to undergo orbital decompression and 5 of them had improvement in vision. All proven ROCM patients were treated with 3 weeks of injection liposomal amphotericin B along with oral posaconazole $300 \mathrm{mg}$ for 3-6 weeks. Post-operative histopathology noted fungal invasion of blood vessels in 19 cases and thrombus in 10 cases of orbital involvement. No mortality was reported in the study group. COVID variant associated thrombosis may be the reason for increased incidence of mucor mycosis in post COVID patients. Team work, appropriate guidelines and adequate supply of medications helped in achieving desired outcome.
\end{abstract}

Keywords: ROCM, Post COVID, Mucormycosis

\section{INTRODUCTION}

COVID-19 has been a global pandemic since November 2019 and has infected close to 200 million people worldwide. ${ }^{1}$ Mucorales of zygomycetes family of fungus causes life threatening infections in immune compromised patients. ${ }^{2}$ In 2019, Cornley et al published the global guidelines for management of mucormycosis. ${ }^{3}$

Rhino orbito cerebral mucormycosis (ROCM) infection occurring in post COVID-19 patients was an unexpected challenge. From May 2021 in our institution we have seen an increase in number of mucormycosis patients among COVID-19 recovered patients. We setup a separate ward for the treatment of these patients to enable in multidisciplinary monitoring. The team consisted of physician, otolaryngologist, ophthalmologist, microbiologist, pathologist, dentist, radiologist and anaesthesiologist.

In Tamil Nadu, India directorate of medical education published management guidelines for mucor mycosis in May 2021. ${ }^{4}$

According to this guidelines annexure 4, ROCM shall be staged as following. ${ }^{4}$ 
Stage 1 includes involvement of nasal mucosa alone; stage 2 includes extension to paranasal sinuses; stage 3 includes extending to orbit and stage 4 includes intracranial extension (Figure 1).
The management of ROCM was based on classifying the possible ROCM patients with further investigations as ROCM unlikely, probable ROCM or proven ROCM. We have followed this algorithm in management of our patients (Figure 2).

\section{Proposed Staging of Rhino-Orbito-Cerebral Mucormycosis (ROCM)}

\begin{tabular}{|c|c|c|c|c|}
\hline $\begin{array}{l}\text { Staging of Rhino-Orbito-Cerebral } \\
\text { Mucormycosis }\end{array}$ & Symptoms & Signs & $\begin{array}{l}\text { Primary } \\
\text { Assessment }\end{array}$ & $\begin{array}{l}\text { Confirmation of } \\
\text { Diagnosis }\end{array}$ \\
\hline $\begin{array}{l}\text { Stage 1: Involvement of the nasal } \\
\text { mucosa } \\
\text { 1a: Limited to the middle turbinate } \\
\text { 1b: Involvement of the inferior } \\
\text { turbinate or ostium of the } \\
\text { nasolacrimal duct } \\
\text { 1c: Involvement of the nasal septum } \\
\text { 1d: Bilateral nasal mucosal } \\
\text { involvement }\end{array}$ & $\begin{array}{l}\text { Nasal stuffiness, } \\
\text { nasal discharge, } \\
\text { foul smell, } \\
\text { epistaxis }\end{array}$ & $\begin{array}{l}\text { Foul-smelling sticky mucoid } \\
\text { or black-tinged, or granular } \\
\text { or haemorrhagic nasal } \\
\text { discharge, nasal mucosal } \\
\text { inflammation, erythema, } \\
\text { violaceous or blue } \\
\text { discoloration, pale ulcer, } \\
\text { anaesthesia, ischemia, } \\
\text { eschar }\end{array}$ & $\begin{array}{l}\text { Diagnostic nasal } \\
\text { endoscopy, } \\
\text { Contrast- } \\
\text { enhanced MRI } \\
\text { (preferred) or CT- } \\
\text { scan }\end{array}$ & $\begin{array}{l}\text { Deep nasal swab } \\
\text { or endoscopy- } \\
\text { guided nasal swab } \\
\text { or nasal mucosal } \\
\text { biopsy for direct } \\
\text { microscopy, culture } \\
\text { and molecular } \\
\text { diagnostics; nasal } \\
\text { mucosal biopsy for } \\
\text { rapid } \\
\text { histopathology with } \\
\text { special stains }\end{array}$ \\
\hline $\begin{array}{l}\text { Stage 2: Involvement of paranasal } \\
\text { sinuses } \\
\text { 2a: One sinus } \\
\text { 2b: Two ipsilateral sinuses } \\
\text { 2c: > Two ipsilateral sinuses and/or } \\
\text { palate/oral cavity } \\
\text { 2d: Bilateral paranasal sinus } \\
\text { involvement or involvement of the } \\
\text { zygoma or mandible }\end{array}$ & $\begin{array}{l}\text { Symptoms in } \\
\text { Stage } 1+\text { facial } \\
\text { pain, facial edema, } \\
\text { dental pain, } \\
\text { systemic } \\
\text { symptoms } \\
\text { (malaise, fever) }\end{array}$ & $\begin{array}{l}\text { Signs in Stage } 1+ \\
\text { unilateral or bilateral, } \\
\text { localized or diffuse facial } \\
\text { edema, edema localized } \\
\text { over the sinuses, localized } \\
\text { sinus tenderness }\end{array}$ & $\begin{array}{l}\text { Diagnostic nasal } \\
\text { endoscopy, } \\
\text { Contrast- } \\
\text { enhanced MRI } \\
\text { (preferred) or CT- } \\
\text { scan }\end{array}$ & $\begin{array}{l}\text { Same as Stage } 1+ \\
\text { sinus biopsy for } \\
\text { direct microscopy, } \\
\text { culture and } \\
\text { molecular } \\
\text { diagnostics and } \\
\text { rapid } \\
\text { histopathology }\end{array}$ \\
\hline $\begin{array}{l}\text { Stage } 3 \text { : Involvement of the orbit } \\
\text { 3a: Nasolacrimal duct, medial orbit, } \\
\text { vision unaffected } \\
\text { 3b: Diffuse orbital involvement ( }>1 \\
\text { quadrant or }>2 \text { structures), vision } \\
\text { unaffected } \\
\text { 3c: Central retinal artery or } \\
\text { ophthalmic artery occlusion or } \\
\text { superior ophthalmic vein } \\
\text { thrombosis; involvement of the } \\
\text { superior orbital fissure, inferior } \\
\text { orbital fissure, orbital apex, loss } \\
\text { of vision } \\
\text { 3d: Bilateral orbital involvement }\end{array}$ & $\begin{array}{l}\text { Symptoms in } \\
\text { Stage } 1 \text { and } 2+ \\
\text { pain in the eye, } \\
\text { proptosis, ptosis, } \\
\text { diplopia, loss of } \\
\text { vision, infraorbital } \\
\text { and facial V1 V2 } \\
\text { nerve anesthesia }\end{array}$ & $\begin{array}{l}\text { Signs in Stage } 1 \text { and } 2+ \\
\text { conjunctival chemoses, } \\
\text { isolated ocular motility } \\
\text { restriction, ptosis, } \\
\text { proptosis, infraorbital nerve } \\
\text { anesthesia, central retinal } \\
\text { artery occlusion, features of } \\
\text { ophthaimic artery occlusion } \\
\text { and superior ophthalmic } \\
\text { vein thrombosis. V1 and V2 } \\
\text { nerve anesthesia, and } \\
\text { features of III, IV and VI } \\
\text { nerve palsy indicating } \\
\text { orbital apex/superior orbital } \\
\text { fissure involvement. }\end{array}$ & $\begin{array}{l}\text { Diagnostic nasal } \\
\text { endoscopy, } \\
\text { Contrast- } \\
\text { enhanced MRI } \\
\text { (preferred) or CT- } \\
\text { scan }\end{array}$ & $\begin{array}{l}\text { Same as Stage } 2+ \\
\text { orbital biopsy if } \\
\text { indicated and if } \\
\text { feasible (if the } \\
\text { disease is } \\
\text { predominantly } \\
\text { orbital) for direct } \\
\text { microscopy, culture } \\
\text { and molecular } \\
\text { diagnostics and } \\
\text { rapid } \\
\text { histopathology }\end{array}$ \\
\hline $\begin{array}{l}\text { Stage 4: Involvement of the CNS } \\
\text { 4a: Focal or partial cavernous sinus } \\
\text { involvement and/or involvement } \\
\text { of the cribriform plate } \\
\text { 4b: Diffuse cavernous sinus } \\
\text { involvement and/or cavernous } \\
\text { sinus thrombosis } \\
\text { 4c: Involvement beyond the } \\
\text { cavernous sinus, involvement } \\
\text { of the skull base, internal carotid } \\
\text { artery occlusion, brain infarction } \\
\text { 4d: Multifocal or diffuse CNS disease }\end{array}$ & $\begin{array}{l}\text { Symptoms in } \\
\text { Stage } 1 \text { to } 3+ \\
\text { bilateral proptosis, } \\
\text { paralysis, altered } \\
\text { consciousness, } \\
\text { focal seizures }\end{array}$ & $\begin{array}{l}\text { Signs in Stage } 1-3 \text { (some } \\
\text { features overlap with Stage } \\
3 \text { ) + V1 and V2 nerve } \\
\text { anesthesia, ptosis, and } \\
\text { features of III, IV and VI } \\
\text { nerve palsy indicate } \\
\text { cavernous sinus } \\
\text { involvement. Bilaterality of } \\
\text { these signs with } \\
\text { contralateral orbital edema } \\
\text { with no clinico-radiological } \\
\text { evidence of paranasal } \\
\text { sinus or orbital involvement } \\
\text { on the contralateral side } \\
\text { indicate cavernous sinus } \\
\text { thrombosis. Hemiparesis, } \\
\text { altered consciousness and } \\
\text { focal seizures indicate } \\
\text { brain invasion and } \\
\text { infarction. }\end{array}$ & $\begin{array}{l}\text { Diagnostic } \\
\text { endoscopy, } \\
\text { Contrast- } \\
\text { enhanced CT } \\
\text { Scan, MRI } \\
\text { (preferred) }\end{array}$ & Same as Stage 3 \\
\hline
\end{tabular}

Figure 1: Proposed staging of POSTCOVID ROCM suggested by guidelines of DME, Government of Tamil Nadu, India. 


\section{Management Approach for Possible, Probable or Proven Rhino-Orbito-Cerebral Mucormycosis (ROCM)}

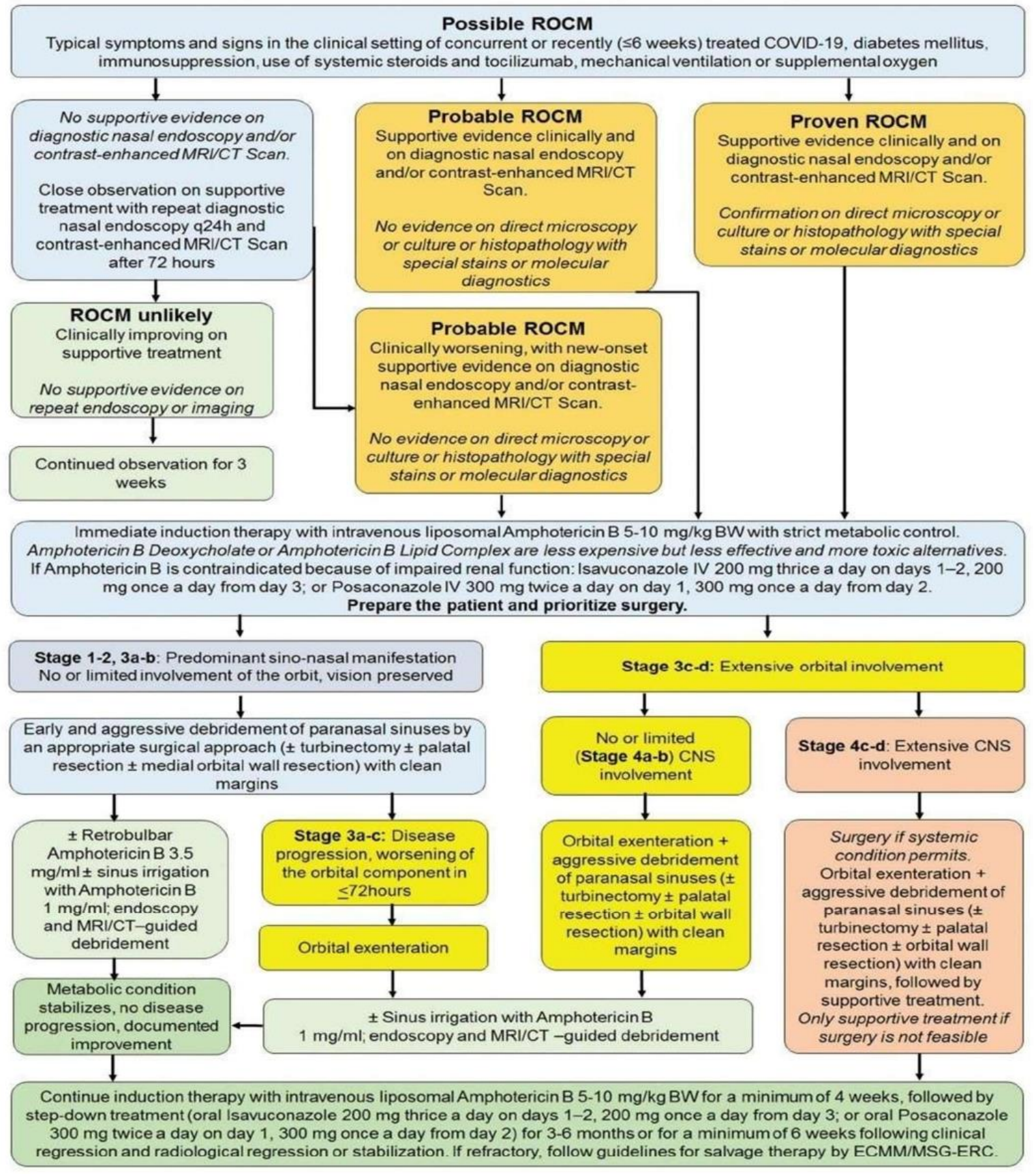

Figure 2: Management algorithm for POSTCOVID ROCM suggested by guidelines of DME, Government of Tamil Nadu, India.

\section{CASE SERIES}

All patients who presented after COVID infection treatment with suspected symptoms of ROCM like nasal obstruction, nasal discharge, visual loss, protrusion of eyeball were admitted in mucor ward.

We had 30 patients of possible ROCM in mucor ward so far from May 2021 (Table 1). There were 19 (64\%) male and $11(36 \%)$ female patients. Age varied between 18 years to 82 years. Mean age was 56 years. $24(80 \%)$ patients had diabetes along with other diseases, $6(20 \%)$ patients had no predisposing factors. $21(70 \%)$ patients were oxygen dependent during COVID treatment.

Initial assessment classifying them as possible ROCM will undergo routine investigations along with radiology, smear for microbiology and biopsy for pathology through 
diagnostic nasal endoscopy (Figure 3) and started with antifungal depending on clinical suspicion of ROCM.

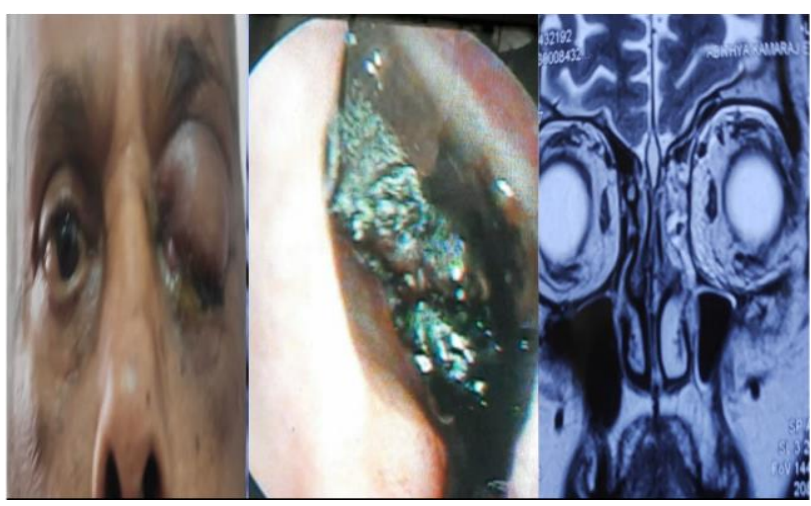

Figure 3: Patient with proptosis (a) proptosis left eye, (b) black middle turbinate, and (c) MRI sinus with orbit.

A magnetic resonance imaging (MRI) para nasal sinus with orbit is the preferred investigation of choice. Radiologists report the cases in prescribed format (Figure 4) staging ROCM. In stage 1 (36\%), nasal cavity alone was involved in 11 patients. In stage $2(28 \%)$, paranasal sinus extension was seen in 8 patients. In stage $3(33 \%)$, orbital extension was seen in 10 patients; and in stage 4 $(3 \%)$, intra cranial extension was seen in 1 patient.
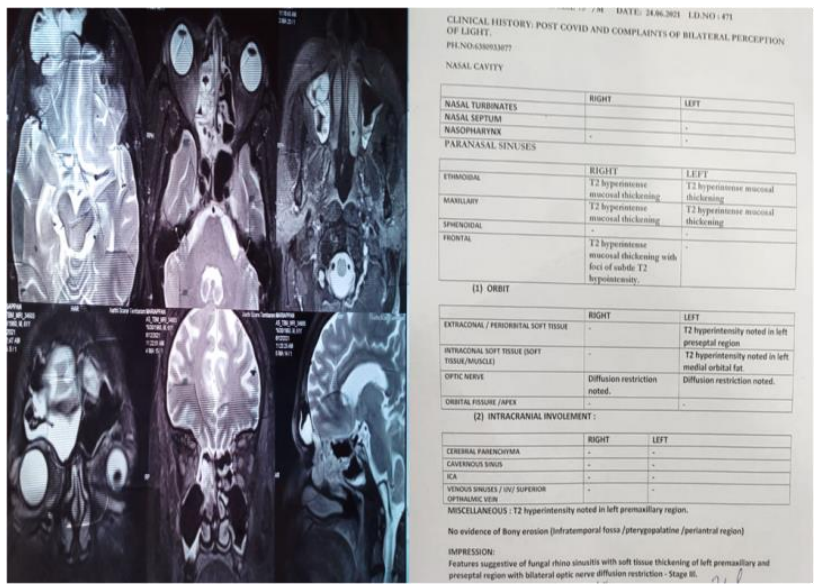

Figure 4: MRI paranasal sinuses with orbit report with staging of ROCM.

Smear for microbiology staining and culture was sent in sterile container and biopsy for histopathology was sent in formalin with clinical details.

Patients with no microbiological or histopathological evidence of mucor were grouped as probable ROCM (33\%). In 8 patients of probable ROCM, only medical management with oral antifungal tablet posaconazole 300 $\mathrm{mg}$ once a day initially was given till confirmation of diagnosis then observed with regular nasal endoscopy for new symptoms.
Smear examination and culture proved mucor in 13 cases (43\%). Histopathology examination (Figure 5A) confirmed invasive fungal infection in 19 cases and 1 non-invasive fungal infection, and they were grouped as proven ROCM was 20 patients (67\%).

Upon confirmation of either or both patients will be taken up for early endoscopic debridement and orbital decompression if needed.

20 patients of proven ROCM and 2 probable ROCM underwent surgery. Endoscopic debridement alone was done in 10 patients (50\%) and endoscopic orbital decompression was done in 10 patients $(50 \%)$ along with debridement. 6 patients $(30 \%)$ had bilateral disease. 4 patients $(20 \%)$ underwent repeat debridement. 3 patients (15\%) had palatal debridement. 1patient had intracranial extension was managed with endoscopic debridement and drainage. The progress recorded and condition at the time of discharge noted.

All proven ROCM completed 3 weeks of injection liposomal amphotericin B along with oral posaconazole $300 \mathrm{mg}$ for 3-6 weeks.

Post op debrided material was sent for histopathological examination. Post-operative histopathology (Figure 5B) report showed blood vessels invaded with fungus in 19 cases of proven ROCM hence termed as invasive fungal sinusitis, except 1 case which was non-invasive fungal sinusitis. In 10 cases of stage 3 proven ROCM having orbital involvement, along with angio invasion of fungus, thrombi noted in blood vessels (Figure 5C).

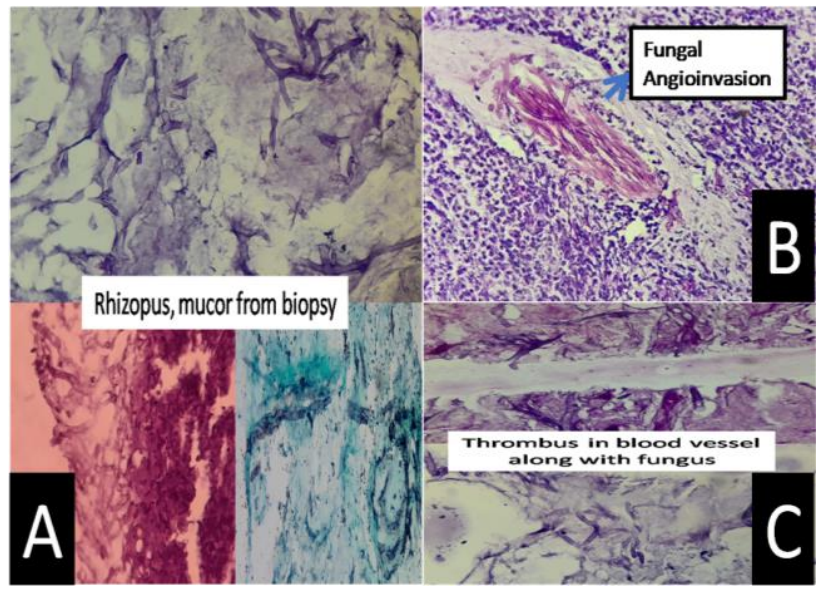

Figure 5: Histopathology picture.

$21(70 \%)$ patients had successful outcome. 8 (27\%) patients had complete cure and $13(43 \%)$ patients had improvement in symptoms. $9(30 \%)$ patients had no worsening of symptoms or remained as same even after treatment. In 10 patients who underwent orbital decompression, vision improvement was there in $5(50 \%)$ patients. The mean hospital stay was 14 days for ROCM. 
Table 1: Master chart.

\begin{tabular}{|c|c|c|c|c|c|c|c|c|c|c|c|c|c|}
\hline $\begin{array}{l}\text { S. } \\
\text { no. }\end{array}$ & Age & Sex & $\begin{array}{l}\text { Predisposing } \\
\text { factors }\end{array}$ & $\begin{array}{l}\text { COVID } \\
\text { severity }\end{array}$ & Symptoms & Staging & Micro & Biopsy & Surgical extent & $\begin{array}{l}\text { Final } \\
\text { diagnosis }\end{array}$ & Antifungals & $\begin{array}{l}\text { Condition on } \\
\text { discharge }\end{array}$ & $\begin{array}{l}\text { Duration } \\
\text { of stay } \\
\text { (days) }\end{array}$ \\
\hline 1 & 47 & M & DM/SHT & $\begin{array}{l}\text { Oxygen } \\
\text { dependent }\end{array}$ & $\begin{array}{l}\text { Proptosis, vision } \\
\text { loss, nasal discharge }\end{array}$ & 3 & Mucor & $\begin{array}{l}\text { Invasive } \\
\text { fungal }\end{array}$ & $\begin{array}{l}\text { Endoscopic orbit, } \\
\text { decompression }\end{array}$ & $\begin{array}{l}\text { Proven } \\
\text { ROCM }\end{array}$ & $\begin{array}{l}\text { Ampho, } \\
\text { Posco }\end{array}$ & $\begin{array}{l}\text { Vision } \\
\text { improved }\end{array}$ & 16 \\
\hline 2 & 68 & $\mathrm{~F}$ & DM/IHD & $\begin{array}{l}\text { Oxygen } \\
\text { dependent }\end{array}$ & $\begin{array}{l}\text { Proptosis, vision } \\
\text { loss, palate, nasal } \\
\text { discharge }\end{array}$ & 3 & Mucor & $\begin{array}{l}\text { Invasive } \\
\text { fungal }\end{array}$ & $\begin{array}{l}\text { Endoscopic orbit, } \\
\text { decompression, } \\
\text { repeat palate, } \\
\text { debridement }\end{array}$ & $\begin{array}{l}\text { Proven } \\
\text { ROCM }\end{array}$ & $\begin{array}{l}\text { Ampho, } \\
\text { Posco }\end{array}$ & $\begin{array}{l}\text { No worsening } \\
\text { of symptoms }\end{array}$ & 30 \\
\hline 3 & 82 & M & DM/SHT/IHD & $\begin{array}{l}\text { Oxygen } \\
\text { dependent }\end{array}$ & $\begin{array}{l}\text { Proptosis, vision } \\
\text { loss, nasal discharge }\end{array}$ & 3 & Mucor & $\begin{array}{l}\text { Invasive } \\
\text { fungal }\end{array}$ & $\begin{array}{l}\text { Endoscopic orbit, } \\
\text { decompression }\end{array}$ & $\begin{array}{l}\text { Proven } \\
\text { ROCM }\end{array}$ & $\begin{array}{l}\text { Ampho, } \\
\text { Posco }\end{array}$ & $\begin{array}{l}\text { Vision } \\
\text { improved }\end{array}$ & 25 \\
\hline 4 & 56 & M & $\mathrm{DM}$ & $\begin{array}{l}\text { COVID care } \\
\text { centre }\end{array}$ & $\begin{array}{l}\text { Nasal discharge, left } \\
\text { cheek pain }\end{array}$ & 2 & Mucor & $\begin{array}{l}\text { Invasive } \\
\text { fungal }\end{array}$ & $\begin{array}{l}\text { Endoscopic orbit, } \\
\text { decompression }\end{array}$ & $\begin{array}{l}\text { Proven } \\
\text { ROCM }\end{array}$ & $\begin{array}{l}\text { Ampho, } \\
\text { Posco }\end{array}$ & Cured & 14 \\
\hline 5 & 56 & M & $\mathrm{DM}$ & $\begin{array}{l}\text { Oxygen } \\
\text { dependent }\end{array}$ & $\begin{array}{l}\text { Proptosis, vision } \\
\text { loss, nasal discharge }\end{array}$ & 3 & Mucor & $\begin{array}{l}\text { Invasive } \\
\text { fungal }\end{array}$ & $\begin{array}{l}\text { Endoscopic orbit, } \\
\text { decompression }\end{array}$ & $\begin{array}{l}\text { Proven } \\
\text { ROCM }\end{array}$ & $\begin{array}{l}\text { Ampho, } \\
\text { Posco }\end{array}$ & $\begin{array}{l}\text { Vision } \\
\text { improved }\end{array}$ & 12 \\
\hline 6 & 44 & $\mathrm{~F}$ & $\mathrm{DM}$ & $\begin{array}{l}\text { Oxygen } \\
\text { dependent }\end{array}$ & Nasal discharge & 1 & Nil & Nil & Not done & $\begin{array}{l}\text { Probable } \\
\text { ROCM }\end{array}$ & Posco & Improved & 10 \\
\hline 7 & 65 & $\mathrm{~F}$ & $\mathrm{DM} / \mathrm{SHT}$ & $\begin{array}{l}\text { Oxygen } \\
\text { dependent }\end{array}$ & $\begin{array}{l}\text { Proptosis, vision } \\
\text { loss, palate, nasal } \\
\text { discharge }\end{array}$ & 3 & Mucor & $\begin{array}{l}\text { Invasive } \\
\text { fungal }\end{array}$ & $\begin{array}{l}\text { Endoscopic orbit } \\
\text { Decompression }\end{array}$ & $\begin{array}{l}\text { Proven } \\
\text { ROCM }\end{array}$ & $\begin{array}{l}\text { Ampho } \\
\text { Posco }\end{array}$ & $\begin{array}{l}\text { No worsening } \\
\text { of symptoms }\end{array}$ & 16 \\
\hline 8 & 31 & $\mathrm{~F}$ & Nil & $\begin{array}{l}\text { Home } \\
\text { isolation }\end{array}$ & $\begin{array}{l}\text { Nasal discharge, left } \\
\text { cheek pain }\end{array}$ & 2 & Nil & $\begin{array}{l}\text { Non- } \\
\text { invasive } \\
\text { fungal }\end{array}$ & $\begin{array}{l}\text { Endoscopic } \\
\text { debridement }\end{array}$ & $\begin{array}{l}\text { Probable } \\
\text { ROCM }\end{array}$ & Posco & Cured & 10 \\
\hline 9 & 37 & $\mathrm{~F}$ & $\mathrm{DM}$ & $\begin{array}{l}\text { Oxygen } \\
\text { dependent }\end{array}$ & Nasal discharge & 1 & Nil & Nil & $\begin{array}{l}\text { Endoscopic } \\
\text { debridement }\end{array}$ & $\begin{array}{l}\text { Probable } \\
\text { ROCM }\end{array}$ & Posco & Status same & 14 \\
\hline 10 & 48 & $\mathrm{~F}$ & $\mathrm{DM}$ & $\begin{array}{l}\text { Oxygen } \\
\text { dependent }\end{array}$ & Nasal discharge & 1 & Nil & Nil & Not done & $\begin{array}{l}\text { Probable } \\
\text { ROCM }\end{array}$ & Posco & Improved & 14 \\
\hline 11 & 63 & M & $\mathrm{DM}$ & $\begin{array}{l}\text { Oxygen } \\
\text { dependent }\end{array}$ & $\begin{array}{l}\text { Proptosis } \\
\text { Vision loss } \\
\text { Nasal discharge } \\
\text { Seizures }\end{array}$ & 4 & Mucor & $\begin{array}{l}\text { Invasive } \\
\text { fungal }\end{array}$ & $\begin{array}{l}\text { Endoscopic orbit, } \\
\text { decompression, } \\
\text { repeat debridement }\end{array}$ & $\begin{array}{l}\text { Proven } \\
\text { ROCM }\end{array}$ & $\begin{array}{l}\text { Ampho } \\
\text { Posco }\end{array}$ & $\begin{array}{l}\text { Vision } \\
\text { improved, } \\
\text { seizures } \\
\text { stopped }\end{array}$ & 25 \\
\hline 12 & 57 & M & DM/SHT & $\begin{array}{l}\text { Oxygen } \\
\text { dependent }\end{array}$ & $\begin{array}{l}\text { Proptosis } \\
\text { Vision loss } \\
\text { Nasal discharge }\end{array}$ & 3 & Mucor & $\begin{array}{l}\text { Invasive } \\
\text { fungal }\end{array}$ & $\begin{array}{l}\text { Endoscopic orbit, } \\
\text { decompression }\end{array}$ & $\begin{array}{l}\text { Proven } \\
\text { ROCM }\end{array}$ & $\begin{array}{l}\text { Ampho } \\
\text { Posco }\end{array}$ & $\begin{array}{l}\text { No worsening } \\
\text { of symptoms }\end{array}$ & 16 \\
\hline 13 & 55 & M & Nil & Ward & Nasal discharge & 1 & Nil & Nil & Not done & $\begin{array}{l}\text { Probable } \\
\text { ROCM }\end{array}$ & Posco & Improved & 7 \\
\hline 14 & 37 & $\mathrm{~F}$ & $\mathrm{DM}$ & $\begin{array}{l}\text { Oxygen } \\
\text { dependent }\end{array}$ & Nasal discharge & 1 & Nil & Nil & $\begin{array}{l}\text { Endoscopic } \\
\text { debridement }\end{array}$ & $\begin{array}{l}\text { Probable } \\
\text { ROCM }\end{array}$ & Posco & Status same & 14 \\
\hline 15 & 53 & $\mathrm{~F}$ & Nil & Ward & Nasal discharge & 1 & Nil & Nil & Not done & $\begin{array}{l}\text { Probable } \\
\text { ROCM }\end{array}$ & Posco & Improved & 7 \\
\hline 16 & 82 & M & DM/SHT/IHD & $\begin{array}{l}\text { Oxygen } \\
\text { dependent }\end{array}$ & $\begin{array}{l}\text { Proptosis, vision } \\
\text { loss, nasal discharge }\end{array}$ & 3 & Mucor & $\begin{array}{l}\text { Invasive } \\
\text { fungal }\end{array}$ & $\begin{array}{l}\text { Endoscopic orbit, } \\
\text { decompression }\end{array}$ & $\begin{array}{l}\text { Proven } \\
\text { ROCM }\end{array}$ & $\begin{array}{l}\text { Ampho, } \\
\text { Posco }\end{array}$ & $\begin{array}{l}\text { Vision } \\
\text { improved }\end{array}$ & 25 \\
\hline
\end{tabular}

Continued. 


\begin{tabular}{|c|c|c|c|c|c|c|c|c|c|c|c|c|c|}
\hline $\begin{array}{l}\text { S. } \\
\text { no. }\end{array}$ & Age & Sex & $\begin{array}{l}\text { Predisposing } \\
\text { factors }\end{array}$ & $\begin{array}{l}\text { COVID } \\
\text { severity }\end{array}$ & Symptoms & Staging & Micro & Biopsy & Surgical extent & $\begin{array}{l}\text { Final } \\
\text { diagnosis }\end{array}$ & Antifungals & $\begin{array}{l}\text { Condition on } \\
\text { discharge }\end{array}$ & $\begin{array}{l}\text { Duration } \\
\text { of stay } \\
\text { (days) }\end{array}$ \\
\hline 17 & 61 & M & $\mathrm{Dm} / \mathrm{sht}$ & $\begin{array}{l}\text { Oxygen } \\
\text { dependent }\end{array}$ & $\begin{array}{l}\text { Proptosis, vision } \\
\text { loss, nasal discharge }\end{array}$ & 3 & Mucor & $\begin{array}{l}\text { Invasive } \\
\text { fungal }\end{array}$ & $\begin{array}{l}\text { Endoscopic orbit, } \\
\text { decompression }\end{array}$ & $\begin{array}{l}\text { Proven } \\
\text { ROCM }\end{array}$ & $\begin{array}{l}\text { Ampho, } \\
\text { Posco }\end{array}$ & $\begin{array}{l}\text { No worsening } \\
\text { of symptoms }\end{array}$ & 16 \\
\hline 18 & 45 & M & DM & Ward & Nasal discharge & 1 & Nil & Nil & Not done & $\begin{array}{l}\text { Probable } \\
\text { ROCM }\end{array}$ & Posco & Improved & 14 \\
\hline 19 & 75 & $\mathrm{M}$ & DM/IHD & $\begin{array}{l}\text { Oxygen } \\
\text { dependent }\end{array}$ & $\begin{array}{l}\text { Proptosis, vision } \\
\text { loss, nasal discharge }\end{array}$ & 3 & Mucor & $\begin{array}{l}\text { Invasive } \\
\text { fungal }\end{array}$ & $\begin{array}{l}\text { Endoscopic orbit, } \\
\text { decompression }\end{array}$ & $\begin{array}{l}\text { Proven } \\
\text { ROCM }\end{array}$ & $\begin{array}{l}\text { Ampho, } \\
\text { Posco }\end{array}$ & $\begin{array}{l}\text { No worsening } \\
\text { of symptoms }\end{array}$ & 16 \\
\hline 20 & 54 & $\mathrm{M}$ & $\mathrm{DM}$ & $\begin{array}{l}\text { COVID care } \\
\text { centre }\end{array}$ & $\begin{array}{l}\text { Cheek pain, nasal } \\
\text { discharge }\end{array}$ & 2 & Nil & $\begin{array}{l}\text { Invasive } \\
\text { fungal }\end{array}$ & $\begin{array}{l}\text { Endoscopic } \\
\text { debridement }\end{array}$ & $\begin{array}{l}\text { Proven } \\
\text { ROCM }\end{array}$ & $\begin{array}{l}\text { Ampho, } \\
\text { Posco }\end{array}$ & Cured & 16 \\
\hline 21 & 32 & M & DM & $\begin{array}{l}\text { Oxygen } \\
\text { dependent }\end{array}$ & $\begin{array}{l}\text { Cheek pain, nasal } \\
\text { discharge }\end{array}$ & 2 & Nil & $\begin{array}{l}\text { Invasive } \\
\text { fungal }\end{array}$ & $\begin{array}{l}\text { Endoscopic } \\
\text { debridement }\end{array}$ & $\begin{array}{l}\text { Proven } \\
\text { ROCM }\end{array}$ & $\begin{array}{l}\text { Ampho, } \\
\text { Posco }\end{array}$ & Cured & 14 \\
\hline 22 & 45 & $\mathrm{M}$ & Nil & Ward & Nasal discharge & 1 & Nil & Nil & Not done & $\begin{array}{l}\text { Probable } \\
\text { ROCM }\end{array}$ & Posco & Improved & 7 \\
\hline 23 & 35 & $\mathrm{M}$ & Nil & $\begin{array}{l}\text { Oxygen } \\
\text { dependent }\end{array}$ & $\begin{array}{l}\text { Cheek pain, nasal } \\
\text { discharge }\end{array}$ & 2 & Nil & $\begin{array}{l}\text { Invasive } \\
\text { fungal }\end{array}$ & $\begin{array}{l}\text { Endoscopic } \\
\text { debridement }\end{array}$ & $\begin{array}{l}\text { Proven } \\
\text { ROCM }\end{array}$ & $\begin{array}{l}\text { Ampho, } \\
\text { Posco }\end{array}$ & Cured & 14 \\
\hline 24 & 53 & $\mathrm{M}$ & DM & $\begin{array}{l}\text { Oxygen } \\
\text { dependent }\end{array}$ & $\begin{array}{l}\text { Cheek pain, nasal } \\
\text { discharge }\end{array}$ & 2 & Nil & $\begin{array}{l}\text { Invasive } \\
\text { fungal }\end{array}$ & $\begin{array}{l}\text { Endoscopic } \\
\text { debridement }\end{array}$ & $\begin{array}{l}\text { Proven } \\
\text { ROCM }\end{array}$ & $\begin{array}{l}\text { Ampho, } \\
\text { Posco }\end{array}$ & Cured & 14 \\
\hline 25 & 57 & M & DM & $\begin{array}{l}\text { Oxygen } \\
\text { dependent }\end{array}$ & $\begin{array}{l}\text { Cheek pain, nasal } \\
\text { discharge }\end{array}$ & 2 & Nil & $\begin{array}{l}\text { Invasive } \\
\text { fungal }\end{array}$ & $\begin{array}{l}\text { Endoscopic } \\
\text { debridement }\end{array}$ & $\begin{array}{l}\text { Proven } \\
\text { ROCM }\end{array}$ & $\begin{array}{l}\text { Ampho, } \\
\text { Posco }\end{array}$ & Cured & 14 \\
\hline 26 & 37 & $\mathrm{~F}$ & $\mathrm{DM}$ & $\begin{array}{l}\text { Oxygen } \\
\text { dependent }\end{array}$ & Nasal discharge & 1 & Nil & Nil & $\begin{array}{l}\text { Endoscopic } \\
\text { debridement }\end{array}$ & $\begin{array}{l}\text { Probable } \\
\text { ROCM }\end{array}$ & Posco & Status same & 14 \\
\hline 27 & 54 & M & $\mathrm{DM}$ & Ward & Nasal discharge & 1 & Nil & Nil & Not done & $\begin{array}{l}\text { Probable } \\
\text { ROCM }\end{array}$ & Posco & Improved & 7 \\
\hline 28 & 65 & $\mathrm{~F}$ & $\mathrm{DM}$ & Ward & Nasal discharge & 1 & Nil & Nil & Not done & $\begin{array}{l}\text { Probable } \\
\text { ROCM }\end{array}$ & Posco & Improved & 7 \\
\hline 29 & 48 & F & Nil & $\begin{array}{l}\text { Oxygen } \\
\text { dependent }\end{array}$ & $\begin{array}{l}\text { Cheek pain, nasal } \\
\text { discharge }\end{array}$ & 2 & Nil & $\begin{array}{l}\text { Invasive } \\
\text { fungal }\end{array}$ & $\begin{array}{l}\text { Endoscopic } \\
\text { debridement }\end{array}$ & $\begin{array}{l}\text { Proven } \\
\text { ROCM }\end{array}$ & $\begin{array}{l}\text { Ampho, } \\
\text { Posco }\end{array}$ & Cured & 14 \\
\hline 30 & 18 & $\mathrm{M}$ & $\mathrm{DM}$ & $\begin{array}{l}\text { Oxygen } \\
\text { dependent }\end{array}$ & $\begin{array}{l}\text { Proptosis, vision } \\
\text { loss, nasal discharge }\end{array}$ & 3 & Mucor & $\begin{array}{l}\text { Invasive } \\
\text { fungal }\end{array}$ & $\begin{array}{l}\text { Endoscopic orbit } \\
\text { Decompression }\end{array}$ & $\begin{array}{l}\text { Proven } \\
\text { ROCM }\end{array}$ & $\begin{array}{l}\text { Ampho, } \\
\text { Posco }\end{array}$ & $\begin{array}{l}\text { No worsening } \\
\text { of symptoms }\end{array}$ & 16 \\
\hline
\end{tabular}

DM-Diabetes mellitus, IHD, ischemic heart disease, SHT-systemic hypertension, ROCM-rhinoorbitocerebral mucormycosis, AMPHO-amphotericin B, POSCO-posaconazole

\section{Table 2: Manifestation and management of ROCM.}

Possible ROCM=30

Stage 1=11; stage 2=8; stage 3=10; stage $4=1$

Probable ROCM=10

Medical management

Proven $\mathrm{ROCM}=20$

Fess

Endoscopic debridement

Endoscopic debridement with orbital decompression

10

Successful outcome $=2$

2

Complete cure $=8$

Unsuccessful outcome $=9$

Mortality - nil No worsening $=6$ Symptoms same $=3$ 


\section{DISCUSSION}

Mucorales cause invasive fungal sinusitis in immunosupressed individuals. ${ }^{5}$ In India among general population mucor mycosis incidence was 0.14 cases per 1000 according to Skiada et al. ${ }^{6}$ In 2020, White et al reported $26.7 \%$ of invasive fungal infection in COVID-19 patients. ${ }^{7}$

The reason for increased incidence of mucor mycosis in COVID infected individuals has been the topic of interest not answered so far.

In China, Song et al concluded impaired T cell immunity as pathogenic factor. ${ }^{8}$ Steroids usage was hinted as cause for ROCM by Mehta et al and Chaudhary et al. ${ }^{9,10}$ Neha et al published a case series of 10 patients with invasive mucormycosis in patients with COVID infection and concluded that impairment of barrier defense with steroids and tocilizumab make COVID patients susceptible for mucormycosis. ${ }^{11}$ Ahmadikia et al cited corticosteroid administration may be the reason for ROCM in COVID 19 patients. ${ }^{12}$ Moorthy et al postulated possible peripheral microthrombi as a cause. ${ }^{13}$ Peman et al stated that the COVID virus itself may be a cause. ${ }^{14}$

In past 6 months there have been publications on post COVID ROCM like Sarkar et al, Alekseyev et al, Maini et al and Krishna et al. ${ }^{15-18}$ Mitra et al published 32 documented post ROCM patients and had similar results. ${ }^{19}$ They concluded that judicious COVID treatment protocol, high index of suspicion, close monitoring of high-risk patients and early institution of treatment can prevent case severity and reduce mortality. Sharma et al published case series of 23 patients with post COVID mucormycosis and concluded that uncontrolled diabetes and over-zealous use of steroids are two main factors aggravating the illness, and both of these must be properly checked. ${ }^{20}$

In our study $20 \%$ patients had no predisposing factor like diabetes before contacting COVID infection. 30\% of patients had only mild COVID and was not treated with steroids or oxygen supplement. Blood vessels filled with thrombus is noted in 10 cases of stage 3 orbital ROCM along with fungus. This may hint upon COVID virus itself may be the reason for ROCM possible due to peripheral thrombosis as mentioned in Moorthy et al and Peman et al. ${ }^{13,14}$

In our study (Table 2) high vigilance and team work helped in identifying cases early. Out of 30 possible ROCM with stage 1 radiology findings, 10 cases had negative finding in microbiology and pathology. They were treated as probable ROCM and followed up regularly.

Surgical debridement was done early in proven ROCM 20 cases. In 10 cases with orbital involvement, surgical decompression and antifungals brought back vision in
$50 \%$ of cases. Mortality was nil in our study group. $70 \%$ patients had satisfactory outcome.

\section{CONCLUSION}

Post COVID ROCM is different from invasive fungal sinusitis occurring in other immune compromised patients. COVID variant associated thrombosis may be the reason for increased incidence of mucor mycosis in post COVID patients. A good team work, appropriate guidelines issued by authorities and adequate supply of antifungal medications have helped us to achieve desired outcome so far in the management of post COVID ROCM in our institution.

\section{ACKNOWLEDGEMENTS}

Authors would like to thanks faculties and residents of Government Kilpauk Medical College, Chennai.

Funding: No funding sources

Conflict of interest: None declared

Ethical approval: The study was approved by the Institutional Ethics Committee

\section{REFERENCES}

1. COVID-19 Data Repository by the Center for Systems Science and Engineering (CSSE) at Johns Hopkins University. Available at: https://github.com/CSSEGISandData/COVID-19.

Accessed on 14 August 2021.

2. Spellberg B, Ibrahim AS. Mucormycosis. In: Jameson JL, Fauci AS, Kasper DL, Hauser SL, Longo D, Loscalzo J, editors. Harrison's principles of internal medicine, 20th edition. McGraw-Hill Education, New York. 2018;1538.

3. Cornely OA, Arenz D, Vehreschild JJ, Vehreschild MJGT, Ellinghoff SC, Seidel D. Global guideline for the diagnosis and management of mucormycosis: an initiative of the European Confederation of medical Mycology in cooperation with the Mycoses Study Group Education and Research Consortium. Lancet Infect Dis. 2019;19:40521.

4. Management guidelines Mucormycosis. Directorate of medical education, Tamil Nadu. Available at: https://www.google.com/url?sa=t\&rct=j\&q=\&esrc= $\mathrm{s} \&$ source $=$ web $\& \mathrm{~cd}=\& \mathrm{cad}=\mathrm{rja} \&$ uact $=8 \& \mathrm{ved}=2 \mathrm{ahU}$ KEwiK1-e3yrfzAhX-

wjgGHZZmA1sQFnoECAIQAQ\&url=https $\% 3 \mathrm{~A} \%$ 2F\%2Fwww.niepmd.tn.nic.in $\% 2$ Fdocuments $\% 2 F G$ ovtTNP_090621.pdf\&usg=AOvVaw02_WFXDTSa Ty4dXAhuWxJB. Accessed on 14 August 2021.

5. Ribes JC, Vanover-Sans CL. Zygomycetes in human disease. Clin Microbiol Rev. 2000;13(2):236-301.

6. Skiada A, Pavleas I, Drogari Apiranthitou M. Epidemiology and diagnosis of mucormycosis: an update. J Fungi. 2020;6(4):265. 
7. White PL, Dhillon R, Cordey A, Hughes H, Faggian $\mathrm{F}$, Soni $\mathrm{S}$, et al. A national strategy to diagnose COVID-19 associated invasive fungal disease in the ICU. Clin Infect Dis. 2020;1298.

8. Song G, Liang G, Liu W. Fungal co-infections associated with global COVID-19 pandemic: a clinical and diagnostic perspective from China. Mycopathologia. 2020;185:599-26.

9. Mehta S, Pandey A. Rhino-orbital mucormycosis associated with COVID-19. Cureus. 2000;12(9):10726.

10. Chowdhary A, Tarai B, Singh A, Sharma A. Multidrug-resistant Candida auris infections in critically ill coronavirus disease patients, India, April-July 2020. Emerg Infect Dis. 2020;26(11):2694-6.

11. Mishra N, Mutya VSS, Thomas A, Rai G, Reddy B, Mohanan AA, et al. A case series of invasive mucormycosis in patients with COVID-19 infection. Int J Otorhinolaryngol Head Neck Surg. 2021;7(5):867-70.

12. Ahmadikia K, Hashemi SJ, Khodavaisy S, Getso MI, Alijani N, Badali $\mathrm{H}$, et al. The double-edged sword of systemic corticosteroid therapy in viral pneumonia: A case report and comparative review of influenza-associated mucormycosis versus COVID-19 associated mucormycosis. Mycoses. 2021;64(8):798-808.

13. Moorthy A, Gaikwad R, Krishna S, Hegde R, Tripathi KK, Kale PG, et al. SARS-CoV-2, Uncontrolled Diabetes and Corticosteroids-An Unholy Trinity in Invasive Fungal Infections of the Maxillofacial Region? A Retrospective, Multicentric Analysis. J Maxillofac Oral Surg. 2021;20(3):1-8.
14. Peman J, Gaitan AR, Vidal CG, Salavert M, Ramirez P, Puchades F, et al. Fungal co-infection in COVID-19 patients: should we be concerned? Rev Iberoam Micol. 2020;37(2):41-6.

15. Sarkar S, Gokhale T, Choudhury SS, Deb AK. COVID-19 and orbital mucormycosis. Indian $\mathrm{J}$ Ophthalmol. 2021;69(4):1002-4.

16. Alekseyev K, Didenko L, Chaudhry B. Rhinocerebral mucormycosis and COVID-19 pneumonia. J Med Cases. 2021;12(3):85-9.

17. Maini A, Tomar G, Khanna D, Kini Y, Mehta H, Bhagyasree V. Sino-orbital mucormycosis in a COVID-19 patient: A case report. Int J Surg Case Rep. 2021;82:105957.

18. Sai Krishna D, Raj H, Kurup P, Juneja M. Maxillofacial Infections in Covid-19 Era-Actuality or the Unforeseen: 2 Case Reports. Indian J Otolaryngol Head Neck Surg. 2021;1-4.

19. Mitra S, Janweja M, Sengupta A. Post-COVID-19 rhino-orbito-cerebral mucormycosis: a new addition to challenges in pandemic control. Eur Arch Otorhinolaryngol. 2021;1-6.

20. Sharma S, Grover M, Bhargava S, Samdani S, Kataria T. Post coronavirus disease mucormycosis: a deadly addition to the pandemic spectrum. J Laryngol Otol. 2021;135(5):442-7.

Cite this article as: Shanthimalar R, Muthuchitra S, Mary NS, Thamizharasan P, Udhayachandrika G, Balaji C, et al. Comprehensive study on manifestation, management and outcome of post COVID rhino orbito cerebral mucormycosis in our institution. Int J Otorhinolaryngol Head Neck Surg 2021;7:1799-806. 